[12] Journ. Path. and Bact., 1913, 18, p, 169.

[13] Jour. Dy. Se., 1925, 8, p. 344-369.

[14] Jour. Dy. Sc., 1923, 6, p. 532.

[15] Loc. cit. (2), p. 356.

[16] Jour, Dy. Sc., 1926, 9, p. 459.

[17] Carne y Leche, 1935, p. 462-469.

[18] Loc. cit. (7), p. 278.

[19] Cit. (3), 1906.

[20] Rev. in Abst. Bact., 1924, 8, p, 317.

[21] Mitt. Lebensm. Hyg., 1928, 19, p. 89-101.

[22] Ref. Zentralb. f. Bakt., 1936-37, 95.

[23] N.-Y. Med.J., 1915, 102, p. 1073-1083.

[24] Le Lait, 1935, 15, p. 833.

[25] Inspeccion Veterinaria-Sanz Egana, 1935, p. 756.

[26] XIII Internationaler Tierärztlicher Kongress, 1938, p. 1144.

\title{
LE TAUX DE LA VITAMINE C DU LAIT DE VACHE ET SON IMPORTANCE DANS L'ALIMENTATION INFANTILE
}

\author{
par \\ MAURICE GUIGOZ
}

La question des vitamines en pratique alimentaire est depuis longtemps déjà, malheureusement, sortie du domaine purement médical. Le public s'en est emparé avec une précipitation qui n'est pas sans inconvénients. A la religion des microbes a succédé la religion des vitamines. De là sont venus bien des abus regrettables. Récemment, certains auteurs ont, avec raison, relevé les exagérations auxquelles on aboutissait. "Il existe déjà du pain vitaminé, du chocolat vitaminé, des pâtes dentifrices vitaminées, des farines vitaminées, des laits vitaminés, des lotions capillaires vitaminées, des bonbons vitaminés, des caramels vitaminés, des savons vitaminés, une religion des vitamines (HOTTINGER). »

Il est évident que ce problème devait prendre une importance plus grande encore en alimentation infantile. Car, si l'adulte peut, à tout moment, varier son alimentation pour remédier au déséquilibre qu'il constate (ou croit constater), l'enfant, jusqu'à six mois au moins, ne relève que d'une alimentation unique : le lait. L'on étudia done très vite la richesse du lait en vitamines. Si l'on admit de suite que les quãntités des facteurs B1, B2, B3, A, étaient largement suffisantes, le facteur D ne se trouvait que dans le lait d'été, et très peu. Restait le problème de la vitamine C, qui n'est pas encore résolu, Afin, cependant, de parer à tout danger, on conseilla l'adjonction au régime de l'enfant de quelques centimètres cubes de 
jus d'orange ou de citron. Cette mesure de prudence devait rapidement dégénérer. Le public augmenta les doses. On donna aux bébés des rations impressionnantes de jus de fruit. On modifia l'alimentation dans le sens d'une adjonetion de plus en plus rapide de crudités. Pour éviter l'avitaminose C on tomba dans les troubles digestifs. Gaehlinger s'élève contre cette "psychose de la carence des vitamines " et (BAUMANN) écrit : "Il n'est pas toujours logique de donner des jus de fruits acides immédiatement après la tétée ou le biberon. "Et HotTinger : "En tant que pédiâtre, je vois pour le moment plus de troubles digestifs par suite d'une alimentation crue ou d'un régime de fruits anticipé ou exagéré, que par une genèse alimentaire différente : infectieuse, constitutionnelle. 》

Il est cependant évident que l'on ne pourra établir des normes précises, logiques, que le jour ou nous serons exactement renseignés sur tous les points dont l'hygiéniste ou le médecin doivent tenir compte, Or, les travaux parus ces dernières années apportent des conclusions très différentes, quand elles ne sont pas directement opposées. C'est pourquoi il nous a semblé utile de faire le point, en essayant de voir quelles sont les conclusions que l'on peut tirer de l'étude des plus récentes recherehes.

La question posée est celle-ei : La teneur en vitamine $C$ du lait de vache est-elle suffisante pour permettre l'alimentation du nourrisson durant un minimum de six ou huit mois, sans avoir à craindre l'apparition de troubles pouvant être rapportés à une carence ou pré-carence en vitamine $C$ ?

Pour y répondre nous devons résoudre les problèmes suivants :

1. Taux de l'acide ascorbique du lait de femme et du lait de vache.

2. Besoins de l'organisme infantile en vitamine C.

$$
*^{*} *
$$

\section{Taux de l'acide ascorbique du lait de femme et du lait de vache}

Un très grand nombre de recherches ont été entreprises et il semble que chacune d'elle ait donné un résultat différent. Ce fait s'explique en partie par la variation possible de la teneur du lait de vache selon les saisons et selon l'alimentation du bétail.

Mais là, déjà, existent des divergences de vues. Certains estiment que cette variation peut être considérable, d'autres ne lui accordent qu'une importance négligeable.

a) Variation sai sonnière. - La teneur du lait en acide ascorbique serait plus élevée au printemps (CULTRERA et BELLINI), en été (Rohmer, Bezssonoff, Mile Sanders et Stoerr). Pour Buruina 
l'influence saisonnière serait minime, presque inexistante. Il faut remarquer d'ailleurs que les différences données ne sont pas très grandes : maximum, $30 \mathrm{mgr}$; minimum, $25 \mathrm{mgr}$. par litre pour Cultrera et Bellini. La teneur moyenne indiquée par Mme RaNDoIN ne montre aucune différence. Nous pouvons done admettre que la saison n'influe que de façon minime sur la teneur du lait en vitamine $\mathrm{C}$, le maximum étant une perte de $0 \mathrm{mgr} .5 \%$ pour le lait d'hiver.

b) Influence de l'alimentation. - Buruiana, Cultrera et Bellini, Jacobson, Hughes, Rohmer et Bezssoatoff, n'ont pas remarqué qu'une différence dans l'alimentation du bétail influait sur la richesse du lait en acide ascorbique. Par contre, un grand nombre d'auteurs ont constaté une influence parfois considérable (Eckles, Freytag, Hart et Steenbock, Hess, ete.). L'alimentation au foin serait préjudiciable au taux de la vitamine $\mathrm{C}$ du lait. Withmann et Riddel trouvent 7-4,5 fois plus d'acide ascorbique par une alimentation riche en fourrage vert que par une alimentation sèche.

Il semble donc logique d'admettre qu'une plus grande prudence s'impose en hiver, période d'alimentation du bétail en fourrage sec.

D'autres -éléments peuvent encore entrer en ligne de compte et faire baisser le taux de l'acide aseorbique du lait. Le lait des vaches gestantes serait appaurri (NEUweILER). Pratiquement, ce fait ne présente pas d'inconvénient, par suite du mélange des laits livrés au consommateur.

\section{Teneur du lait de vache et du lait de femme en vitamine C}

Nous avons établi ci-dessous le tableau des chiffres donnés par quelques auteurs (d'après NeUweILER, WachHoldder, Mme RANDorn et nos recherches personnelles).

QUANTITÉ, EN MG., D'ACIDE ASCORBIQUE, POUR 100 GR. DE LAIT

Auteurs

Blumenberg $(1) \ldots \ldots \ldots \ldots \ldots$

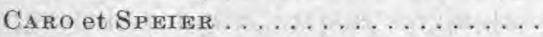

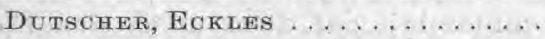

FREYTAG (WACHHOLDER $) \ldots \ldots \ldots \ldots$

Gabathuler

Hart, Stenn Bock, Ellis . . . . . . .

Hess, Unger, Supplee ...........

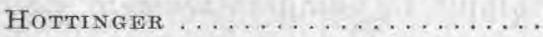

Lait de vache

Lait de femme

$$
\begin{aligned}
& 2 \cdot 2,6 \\
& 0,8 \\
& 2,6-7,5 \\
& 2-3 \text { (été) } \\
& 1 \text { (hiver) } \\
& 3,7 \\
& 1,5-3 \\
& 1,7 \\
& 1,5-3
\end{aligned}
$$

(1) Le tableau de NeuwerLxr indique le nombre de centimètres cubes de lait contenant un 3 dose-protection du cobaye, soit environ $1 \mathrm{mg}$. 5 d'acide aseorbique. Le taux d'aeile ascorbique de 100 grammes de lait a done été culculé par nous sur ces bases. 
vitamine $\mathrm{C}$ suffisante pour lui permettre d'atteindre un certain âge sans accidents, même dans les plus mauvaises conditions.

2. Les variations du taux de vitamine $\mathrm{C}$ du lait de femme sont considérables, et plus grandes qu'avec presque tous les laits d'autre provenance (BAUMANN), - les chiffres extrêmes étant de 0 mgr. 9 $8 \mathrm{mgr} .2 \%$ (BAUMANN). - Il faut done admettre qu'un certain nombre d'enfants nourris au sein reçoivent des quantités de vitamine C qui peuvent être minimes. Or, le scorbut est inconnu ehez les enfants nourris au sein (STEPP, KüHNAU et Schroeder). D'autre part, BAUMANN et RAPPOLT ont remarqué que chez des prématurés nourris constamment avec du lait de femme contenant $1 \mathrm{mgr}$. $2 \mathrm{mgr} .5 \%$ de vitamine $\mathrm{C}$, aucun signe de carence en vitamine $\mathrm{C}$ n'apparut. Ce fait est confirmé par d'autres auteurs (GAHLEN, cité par TOBLER).

Devant les résultats de ces recherches, nous pouvons nous demander si les symptômes de scorbut ou pré-scorbut chez les enfants alimentés artificiellement ne proviennent pas d'une cause autre que la carence du régime en acide ascorbique, cette cause étant cependant liée à l'hypovitaminose C; autrement dit, n'existet-il pas des carences d'origine organique? Toute une série de travaux récents (LECOQ, Mouriquand et son école), nous permettent de répondre affirmativement. Ce sera l'objet d'un prochain travail.

Si nous essayons de voir quelles sont, en milligrammes d'acide ascorbique, les doses que doit recevoir un enfant, journellement, nous nous trouvons en présence de deux groupes, les uns étant partisans de doses élevées, les autres de doses relativement faibles.

Pour les premiers, l'enfant doit recevoir environ 20-30 milligrammes (WIDENBAUER), 25 milligrammes (SzENT-GYÖRGYI, STEPP, SCHROEDER, KING), 25-35 milligrammes à trois mois (NEUWEILER), 20 milligrammes (HESs).

Pour Rietschel, par contre, il existe un véritable fossé entre les recherches théoriques et la clinique. Dans la pratique, des doses, bien inférieures à celles indiquées ci-dessus suffiraient à prévenir toute apparition de signes de carence ou pré-carence. Il se base spécialement sur une expérience de ToBLER faite à Vienne en 1917. Par suite des conditions économiques déplorables, une épidémie de scorbut éclata parmi des enfants réfugiés. Tous les cas furent guéris par la seule adjonction au régime d'une tasse de tisane d'aiguilles de pins. Or, une tasse de cette tisane contiendrait 5-10 milligrammes d'acide ascorbique (Baumann, Rietschel). Il semble done que la quantité de 5 milligrammes de vitamine $\mathrm{C}$ soit celle en-dessous de laquelle un enfant prédisposé au scorbut ne puisse plus être protégé (TOBLER).

Rietschel conclut de l'examen de ces faits : 
Avec un régime ne comportant ni légumes frais ni pommes de terre, il a fallu près d'un an pour que se déclare une épidémie de scorbut.

Une quantité de 5 milligrammes d'acide ascorbique par jour suffisait pour arrêter l'évolution de la maladie et guérir les symptômes les plus graves. Avec 20 ou même 10 à 15 milligrammes la guérison était assurée.

Enfin Rietschen estime qu'il ne faut mettre sur le compte de I'hypovitaminose C, ni les diverses formes de purpura et de thrombopénie, ni les paradentoses et les caries, et que toute la question de la vitamine $\mathrm{C}$ est entièrement à reprendre au point de vue clinique (Analyse de P. E. MorhardT).

Nous constatons done qu'une dose relativement minime d'acide ascorbique suffit pour protéger l'enfant contre le scorbut ou le préscorbut. L'augmentation de l'apport de vitamine C au-dessus de 10-15 milligrammes ne fournit pas une protection plus efficace contre l'infection et n'accélère pas de façon remarquable la croissance de l'enfant (TOBLER).

Le comité d'hygiène de la Société des Nations, pour sa part, estime à 5 milligrammes par jour les besoins de l'enfant en vitamine C.

D'autre part, nous devons enfin rappeler que, pour certains, la vitamine $\mathrm{C}$ agirait à la façon d'un catalyseur, par conséquent aurait une action importante même en quantité infíme (RIETSCHEL). Au surplus, elle peut être re-synthétisée, faisant partie d'un cercle oxydoréducteur (SzENT-GyöRGYI) : ce qui expliquerait qu'il se produise dans l'organisme une re-création constante d'acide ascorbique permettant la diminution de l'apport extérieur. Ce système fonctionnerait relativement longtemps même en l'absence totale de vitamine $\mathrm{C}$ dans l'alimentation (RIETSCHEL).

$$
\text { ** }
$$

\section{Conclusion}

1. L'examen d'un certain nombre d'analyses de lait nous prouve que le lait de femme renferme environ deux fois plus d'acide ascorbique que le lait de vache.

2. Il n'est pas possible de déduire -de ce fait que l'alimentation artificielle ne peut pas fournir à l'enfant la quantité de vitamine C qui lui serait nécessaire.

Un apport maximum de 10-15 milligrammes d'acide ascorbique par jour suffit à empêcher complètement l'apparition de scorbut ou de pré-scorbut. Or, en admettant que le lait de vache contienne environ $2 \mathrm{mgr}$. \% d'acide ascorbique, les besoins de l'enfant sont 
couverts par 500 grammes de lait. Cette quantité est atteinte aux environs du troisième mois, avec de légères différences selon les auteurs (Marfan, Terrien, Renault, Сombe).

Nous avons vu que, quoiqu'il en soit, l'enfant peut, jusqu'à cet âge, se suffïre entièrement à lui-même. Nous pouvons donc dire que, dans l'état actuel de nos connaissances sur la question des vitamines, il est possible d'affirmer :

Le lait de vache contient une quantité suffisante d'acide ascorbique pour assurer la protection certaine de l'enfant sain contre le scorbut ou le pré-scorbut, ou contre.les affections pouvant être rapportées à une avitaminose $C$, et ceci durant tout le temps de l'alimentation lactée exclusive.

\section{BIBLIOGRAPHIE}

(récente ou particulièrement importante)

Gaehlinger. Bruxelles Medical, 23 avril 1939.

Revue médicale de France, mai-juin 1939.

Tobler. Schweiz. Mediz. Woch., 29 juillet 1939.

Mouriquand, M. Dauvergne et Mme Edex. Presse Médicale, 12 août 1939. Rietschel. Klin. Wochensch, 17 décembre 1938.

KROKER. Milchwirtsch. Forschung, no $5,1938$.

Cultrera et Beliini. Ann. chim. applicata, mai 1938.

BaUmann. Schw. Mediz. Woch, 9 octobre 1938.

BaUMANN et RAPPoLD. Zeit. $f$. Vitaminforsch, janvier 1938.

Dutcher, Eckles, Dahle, Mead et Schaeffer. Journal Biol. chem., nº 45 , 1936.

HARRIS et RAY. The Lancet, I, 71, 1935.

Demole. Schw. Mediz. Woch., 18 juillet 1939.

Hess. Cité par Demole.

KIEFERLE. Intern. Milchwirtschafl. Kongrès 1931.

Neuweiler. Die Vitamine der Milch, Huber, 1936.

Neuweiler et Hubscher. Presse médicale, no 37, 1938.

Meunier et Mentzer. Comptes rendus Société biol., no 34,1938 :

Rohmer et Bezssonofy. Presse médicale, no 54, 1934.

WACHнOLDER. Klin. Woch., 1936.

HotTinger. Sehw. Mediz. Woch., no 35, 1939.

Randorn et Simonet. Les vitamines, Paris 1932.

Randorn. Vues actuelles sur le problème de l'alimentation, Paris 1937.

BURUIANA. Le Lait, no $185,1939$.

Farlla. Le Lait, no $185,1939$.

Kon, Watson. Bioch. Journal, no 31, 1937.

Stepp, Kühnau et Schroeder. Die Vitamine und ihre klinische Anwendung, Munich 1938.

Babonneix. Les régimes ehez l'enfant. Masson et Cie, Paris, 1936.

МонR. Deutsch. Mediz. Woch., no 14, 1939.

Sснмrdt. Conf. des vitamines, Moseou, in Bruxelles Medical, no 40, 1939. Bull. Ass, Intern. Pediatrie, LV, no 6, 1937. 
Charles Nrcolte. Dastin des maladies infectieuses.

LECoQ. Déséquilibres alimentaire, Paris, 1938.

(Travail du Service de documentation de la Fabrique suisse des produits au lait Guigoz S. A. Vuadens (Gruyère)).

\title{
LA RÉGLEMENTATION DU LAIT MALPROPRE
}

\author{
par \\ L. HOTON, \\ Docteur ès sciences.
}

Nous sommes tous d'accord sur un point : le lait doit être pur, sain, propre. Laissons de côté les questions relatives à la falsification du lait, mais admettons que si un lait sain est toujours un lait propre, la réciproque n'est pas toujours vraie : la propreté d'un lait, celle qui correspondrait aux exigences d'une réglementation, n'est pas nécessairement le corollaire de la salubrité.

Est-il utile de fixer, par voie de décret, des normes ou des bases analytiques permettant à l'expert de déclarer que tel lait est ou non conforme aux stipulations réglementaires ?

A mon avis, non. La réglementation doit rester, comme on l'a fait en France, dans des généralités; elle doit se borner à exiger que le lait soit recueilli proprement, et qu'il ne soit pas permis de le vendre s'il est coloré, malpropre ou malodorant.

J'exposerai plus loin les raisons qui montrent les dangers d'une définition légale de la propreté du lait.

$\mathrm{Si}$ on parcourt les législations relatives au lait, on constate que sauf une seule exception, les dispositions restent dans les généralités.

L'exception concerne la Belgique. Ce pays avait primitivement édicté en 1894 un règlement qui, à mon sens, était parfait : "il défendait la mise en vente du lait altéré soit..., soit par une tenue défectueuse de l'étable, de la laiterie ou des ustensiles de transport, soit pour toute autre cause telles les manipulations exercées par des personnes peu soigneuses."

Ce règlement est resté en vigueur jusqu'en 1925, c'est alors qu'on a cru devoir indiquer aux experts les eas de souillure qui légalement rendaient le vendeur justiciable des tribunaux.

La Suisse défendait la vente "du lait qui forme un sédiment (1908) \%. Le projet français de 1912 est muet en ce qui concerne la propreté.

En 1915, la Suisse précisait la défense de 1908 en ces termes : "Le lait sera recueilli avec tous les soins de propreté possibles. Destiné à être consommé tel quel, il ne doit pas laisser déposer, 